\title{
Composición y distribución del mesozooplancton en una zona de afloramiento costero (Bahía Culebra, Costa Rica) durante La Niña 1999 y el 2000
}

\author{
Karina Rodríguez-Sáenz ${ }^{1,2}$ \& Alvaro Morales-Ramírez ${ }^{3}$ \\ 1. Sistema de Estudios de Posgrado, Programa Regional de Posgrado en Biología, Universidad de Costa Rica, San Pedro, \\ 11501-2060 San José, Costa Rica. \\ 2. Hábitat Crítico, Asesores Ambientales. Apdo. 389-1300. San José, Costa Rica; karinarodriguez@ racsa.co.cr \\ 3. Centro de Investigación en Ciencias del Mar y Limnología (CIMAR), y Escuela de Biología, Universidad de Costa \\ Rica, San Pedro, 11501-2060 San José, Costa Rica; alvaro.morales@ucr.ac.cr
}

Recibido 12-IX-2011. Corregido 05-II-2012. Aceptado 15-II-2012.

\begin{abstract}
We studied the annual patterns of composition, abundance and spacial-temporal distribution of mesozooplankton in the upwelling zone of Culebra Bay, north Pacific coast of Costa Rica, during the 1999 La Niña event and in 2000. The Bay showed a high variability in physical-chemical conditions during the study period in which 26 mesozooplankton groups (both meroplanktonic and holoplanktonic forms) were found. The copepods (2 358.53 $\left.\pm 2290.90 \mathrm{ind} / \mathrm{m}^{3}\right)$, ostracods ( $\left.1084.46 \pm 1850.20 \mathrm{ind} / \mathrm{m}^{3}\right)$ and invertebrate eggs $(844.26 \pm 7069.27 \mathrm{ind} /$ $\left.\mathrm{m}^{3}\right)$ were the most abundant groups in Bahía Culebra. All mesozooplankton $(0.2-2 \mathrm{~mm})$ data showed high spatial-temporal variability although differences were not significant. Higher abundances were found during 1999, with the exception of appendicularians and crustacean larvae. Higher zooplankton abundances were observed during the dry season of both years. Stations close to the mouth show an oceanic influx of holoplanktonic forms, while inside the bay, the meroplanktonic forms were important. Mesozooplankton abundance and distribution were influenced by coastal upwelling pulses. Rev. Biol. Trop. 60 (Suppl. 2): 143-157. Epub 2012 April 01.
\end{abstract}

Key words: Mesozooplankton, upwelling, La Niña, Gulf of Papagayo, Eastern Pacific, Costa Rica, Culebra Bay.

El zooplancton ocupa una posición clave en las complejas redes tróficas pues transfieren la materia orgánica producida por algas unicelulares, vía fotosíntesis, hasta los niveles tróficos superiores. Los estudios de zooplancton son importantes, ya que a través del análisis de su riqueza específica y composición general, se pueden caracterizar masas de agua o sistemas acuáticos de manera integral y no solo por variables físico-químicas (Suárez-Morales \& Rivera-Arriaga 1998; Morales-Ramírez 2001, 2008). A pesar de la importancia de este grupo de organismos en la ecología de una gran variedad de especies marinas de interés comercial, el conocimiento acerca del zooplancton es muy limitado comparado con lo que se sabe sobre la biodiversidad terrestre de plantas y herbívoros
(Irigoien et al. 2004). Su composición y distribución presenta variaciones espaciales y temporales que son debidas, entre otros, a factores físicos y químicos, tales como la topografía del fondo, la temperatura, la salinidad, el oxígeno disuelto en el agua, y a factores biológicos como la disponibilidad de recursos alimentarios, la capacidad reproductiva, la exclusión competitiva y otros. Se reconoce que dichas variaciones son más complejas en las zonas costeras debido al carácter sinérgico de las influencias océano-continente que allí se presentan (Thurman 1996). Otros factores cuya predicción es aún inexacta, como el fenómeno de El Niño o La Niña, tienen un impacto en la composición y distribución del zooplancton que aún hoy no se tiene claramente establecido. 
Mientas algunos taxones tienden a desaparecer, otros tienden a constituirse en oportunistas que incrementan su abundancia durante estos eventos (Lavaniegos et al. 2002). Se espera, por ejemplo, que como producto del fenómeno de La Niña, se incrementen la biomasa, abundancia y la composición del zooplancton, con respecto a El Niño, para luego llegar a niveles similares a los años en los que no se presenta este tipo de fenómenos.

El zooplancton en el Pacífico costarricense ha sido investigado principalmente en el Golfo de Nicoya (Morales-Ramírez \& VargasZamora 1995, Morales-Ramírez 1996, 2001, von Wangelin \& Wolff 1996, Morales-Ramírez \& Brugnoli-Olivera 2001, Brugnoli-Olivera et al. 2004, Brugnoli-Olivera \& Morales-Ramírez 2008), Golfo Dulce (Morales-Ramírez 1996, 2001, Quesada-Alpízar 2001, Estrada 2003, Morales-Ramírez \& Nowaczyk, 2006, Quesada-Alpízar \& Morales-Ramírez 2006, MoralesRamírez \& Jacob 2008), y en menor grado en el Parque Nacional Isla del Caño (Guzmán \& Obando 1986), Parque Nacional Marino Ballena (Morales-Ramírez en prep.), Bahía Coronado (Morales-Ramírez 1996) y más recientemente en el Parque Nacional Isla del Coco (Morales-Ramírez 2008). Estos estudios se han centrado en aspectos relacionados a la distribución, biomasa, composición y variación temporal y espacial, en ocasiones en presencia de El Niño o en condiciones normales.

El Golfo de Papagayo en el Pacífico Norte de Costa Rica es el tercero en extensión en este país $\left(180 \mathrm{~km}^{2}\right)$ y es mucho más abierto que los golfos de Nicoya y Dulce. Además de su riqueza biológico y pesquera, son conocidos los afloramientos estacionales que ocurren durante la estación seca (diciembre-abril) (NOAA 1979, Bianchi 1991, Fiedler et al. 1991, Brenes et al. 1995, Jiménez 2001a), que provoca claras diferencias de las estaciones en su productividad biológica (Desrosiers et al. 2007). En este golfo se ubica Bahía Culebra, que es una de las bahías más protegida de la influencia del oleaje entre el Golfo de Fonseca (Honduras) y el Golfo de Nicoya (Jiménez 1998). Dentro de esta bahía se desarrollan por lo menos ocho pequeños estuarios con vegetación típica de manglar (Córdoba et al. 1998, Zamora-Trejos \& Cortés 2009), que aportan pequeños caudales a la bahía de forma intermitente y fuerte durante la época lluviosa (obs. pers.). Existen allí comunidades coralinas y corales solitarios únicos en la costa Pacífica del país (Jiménez 1997, 1998, 2001b, Jiménez et al. 2010). De la misma manera se presenta un gran número de especies de peces de arrecife (Dominici-Arosemena et al. 2005) y además es visitada por otras cuantas especies como tortugas marinas, delfines (frecuentemente el manchado, Stenella attenuata) (Rodríguez-Saénz \& RodríguezFonseca 2004).

El único estudio en Bahía Culebra sobre zooplancton ha sido generado por Bednarsky y Morales-Ramírez (2004) quienes investigaron el macrozooplancton en cuatro estaciones dentro y fuera de la bahía y concluyeron que el diverso conjunto de especies de copépodos es un claro indicador de que el sistema está sano. Según este estudio, los copépodos dominan la abundancia del zooplancton de la bahía y varias especies dominantes pertenecen a la familia Eucalanidae, típica de sistemas de afloramiento. Como otros grupos dominantes del zooplancton encontraron los ostrácodos, las salpas y los sifonóforos. Un estudio sobre la diversidad marina de Costa Rica recopila la información de recolectas de sifonóforos y se incluyen 18 especies recolectadas en esta misma área (Rodríguez-Sáenz \& Gasca 2009).

Este estudio tiene como objetivo describir la composición del mesozooplancton de Bahía Culebra y la variación espacio-temporal del grupo en cuatro sitios a lo largo de dos periodos de estación seca y dos de estación lluviosa (1999 y 2000). Además, trata de establecer la posible relación con el fenómeno de La Niña (1999) y el año 2000, basándose en parámetros ambientales como oxígeno disuelto en agua, salinidad, temperatura y profundidad de penetración de la luz (o transparencia de agua). Fueron evaluadas las siguientes hipótesis de trabajo: a) la composición, abundancia y distribución del zooplancton no muestran diferencias significativas en los sitios de muestreo, 
b) los fenómenos a gran escala no afectan la dinámica del zooplancton en Bahía Culebra.

\section{MATERIALES Y MÉTODOS}

Área de estudio: El clima de la región está influenciado por los desplazamientos de la Zona de Convergencia Intertropical. Bajo condiciones "normales", alrededor de diciembre-marzo se desarrolla el fenómeno de afloramiento costero, como producto de la intensificación de los vientos Alisios del Norte y simultáneo a un sistema de alta presión en el Golfo de México y el Caribe (Brenes et al. 1995, Alfaro et al. 2012). La estación lluviosa comienza en mayo, con un breve periodo seco en junio para luego remontar el período de lluvias desde julio-noviembre (Jiménez 1998).

Dentro de esta área se desarrollan pequeños estuarios con vegetación típica de manglar, como los esteros Palmares, Chorizo, Iguanita,
Panamá, entre otros (Córdoba et al. 1998, Zamora-Trejos \& Cortés 2009) que aportan agua dulce a la bahía de forma intermitente pero copiosa durante la época lluviosa. Se ubicaron cuatro puntos de muestreo seleccionados de una forma no aleatoria (Fig. 1). El sitio cuatro (S4) representa la parte más externa y con influencia oceánica (profundidad máxima de 40m); el sitio tres (S3) corresponde al sector de influjo de la bahía (profundidad de $30 \mathrm{~m}$ ); el sitio dos (S2), es la parte central o media de la bahía (profundidad de $20 \mathrm{~m}$ ) y el sitio uno (S1) es la zona con mayor influencia estuarina, por la cercanía con el estero Iguanita (profundidad de 12m).

Muestreo: La toma de muestreas se realizó durante tres meses en la época seca (febrero-mayo) y durante la época lluviosa (setiembre-noviembre) cada 15 días. Se tomaron muestras de agua con una botella Niskin de

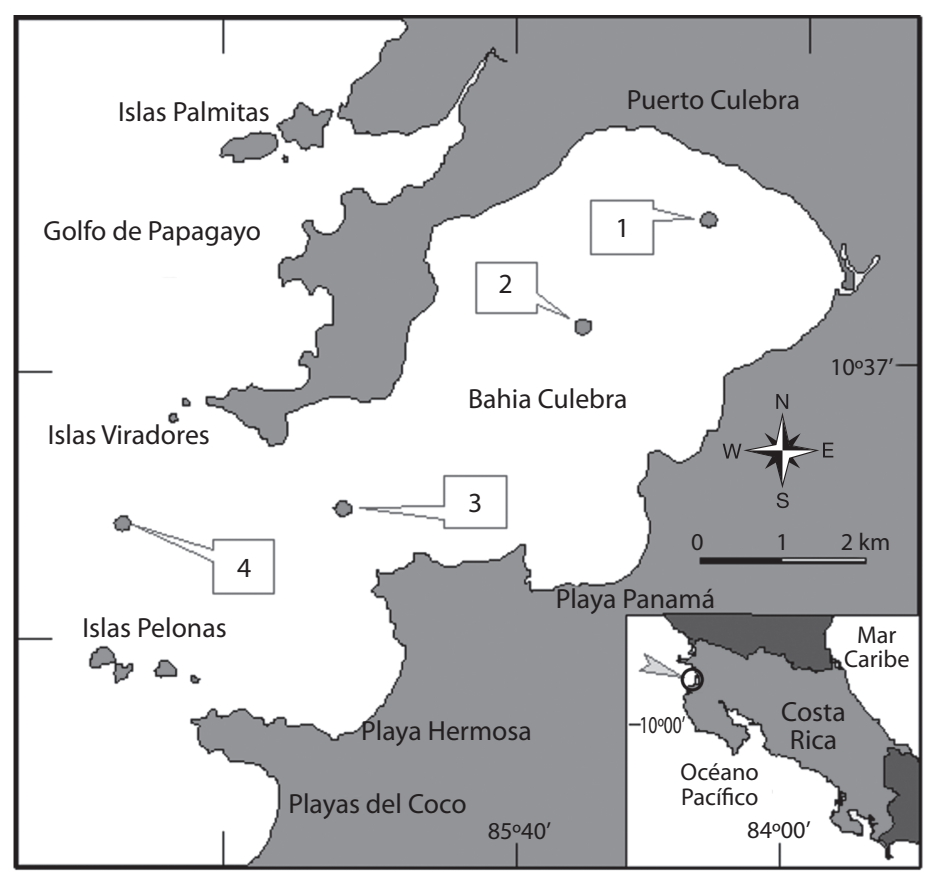

Fig. 1. Ubicación de los sitios de muestreo en Bahía Culebra, Golfo de Papagayo, Pacífico norte de Costa Rica. Modificado de Muller-Parker y Cortés (2001).

Fig. 1. Sampling sites in Bahía Culebra, Gulf of Papagayo, North Pacific of Costa Rica. 
2L, cada $5 \mathrm{~m}$ de profundidad en los sitios $\mathrm{S} 4$, $\mathrm{S} 3$ y $\mathrm{S} 2$ y cada $3 \mathrm{~m}$ en $\mathrm{S} 1$. Para cada muestra se determinó el oxígeno disuelto (mg/l) y la temperatura del agua $\left({ }^{\circ} \mathrm{C}\right)$ con un medidor de oxígeno YSI 52. La salinidad (ups) se midió con un refractómetro de mano ATAGO S/Mill-E; determinó también la profundidad del disco de Secchi. Los muestreos de mesozooplancton se realizaron a bordo de un bote inflable, equipado con un brazo móvil para facilitar su toma. Se utilizó una red cónica de plancton con apertura de poro de $280 \mu \mathrm{m}$ y un diámetro de $47 \mathrm{~cm}$. El tipo de arrastre fue vertical, a lo largo de toda la columna de agua, la cual varió en profundidad según el sitio muestreado. El volumen de agua filtrado se calculó al multiplicar el área de la boca de la red por la distancia recorrida para cada arrastre. Se realizaron observaciones de las condiciones de oleaje, vientos, nubosidad y de condiciones especiales del ambiente, e.g. presencia de proliferaciones algales nocivas.

Métodos: Previo a los muestreos de mesozooplancton se tomaron muestras de agua, cada $5 \mathrm{~m}$ de profundidad (hasta $40 \mathrm{~m}$ en la $\mathrm{S} 4$ y $30 \mathrm{~m}$ en la $\mathrm{S} 3$ ) y cada $3 \mathrm{~m}$ en las $\mathrm{S} 2(18 \mathrm{~m})$ y S1 (15-12m), con una botella Niskin de 2L. Para cada muestra se determinó el oxígeno disuelto (mg/l) y temperatura del agua $\left({ }^{\circ} \mathrm{C}\right)$ con un medidor de oxígeno YSI 52. La salinidad (psu) se midió con un refractómetro de mano ATAGO S/Mill-E y la profundidad a la que dejó de ser visible el disco de Secchi. Además, se realizaron observaciones de las condiciones de oleaje, vientos, nubosidad y otras observaciones especiales como la presencia de mareas rojas, delfines, tortugas, peces muertos.

Una vez tomadas las muestras, fueron fijadas en formalina (4\%, amortiguada con tetraborato de sodio) (Steedman 1976) y trasladadas a los laboratorios del Centro de Investigación en Ciencias del Mar y Limnología (CIMAR) de la Universidad de Costa Rica. Posteriormente, cada muestra fue lavada con agua destilada y fraccionada en un separador de Folsom (Wildco 1831F10) para las clasificaciones taxonómicas y determinación de la abundancia (máxima fracción utilizada 1/64).
Tanto la fracción utilizada como las muestras originales fueron transferidas a alcohol al $70 \%$ para su preservación y almacenamiento. Las abundancias son expresadas en ind. $/ \mathrm{m}^{3}$.

Estadísticas descriptivas (promedio, desviación estándar, ámbito, coeficiente de variación) así como pruebas exploratorias (Análisis de conglomerados, basado en matrices de similitud Bray-Curtis) y confirmatorias (t-student, análisis de variancia de una y dos vías) se utilizaron para analizar los datos con el paquete estadístico SYSTAT 9.0. Los datos de abundancia fueron transformados a $\log 10(\mathrm{x}+1)$ para normalizar las distribuciones y adecuar las varianzas previo al uso de las pruebas de ANOVA. Se tomó un $95 \%$ como límite de confianza para las pruebas confirmatorias. Para realizar el análisis de los datos físico-químicos se utilizó el valor superficial de los diferentes parámetros por sitio de muestreo.

\section{RESULTADOS}

\section{Parámetros ambientales}

Variaciones temporales: Se observó una importante dispersión de los datos con respecto a las fechas de muestreo en los parámetros físicos y químicos, sobre todo durante 1999 (Fig. 2). Aun así, con las comparaciones anuales se determinó que la concentración de oxígeno disuelto en agua fue significativamente mayor durante $1999(6.94 \pm 1.59 \mathrm{mg} / \mathrm{l})$ que durante el año $2000(6.06 \pm 2.09 \mathrm{mg} / \mathrm{l})(\mathrm{t}=2.161, \mathrm{p}=$ 0.033). La salinidad mostró el patrón inverso, de manera que durante 1999 se cuantificó una salinidad significativamente menor (30.74 \pm 2.32PSU) que en el 2000 (32.57 $\pm 1.58 \mathrm{PSU})$ $(\mathrm{t}=4.379, \mathrm{p}=0.001) . \mathrm{La}$ temperatura mostró promedios anuales muy semejantes (1999= $27.84 \pm 1.59$ y $\left.2000=27.66 \pm 1.49^{\circ} \mathrm{C}\right)(\mathrm{t}=0.53$, $\mathrm{p}=0.60)$ de la misma forma que la transparencia del agua $(1999=6.53 \pm 2.11 \mathrm{~m}, 2000=6.88$ $\pm 3.13)(\mathrm{t}=0.41, \mathrm{p}=0.502)$.

Relaciones espaciales: Tanto el oxígeno disuelto, como la salinidad y la transparencia del agua mostraron una misma tendencia con respecto a los sitios de estudio, de manera que 

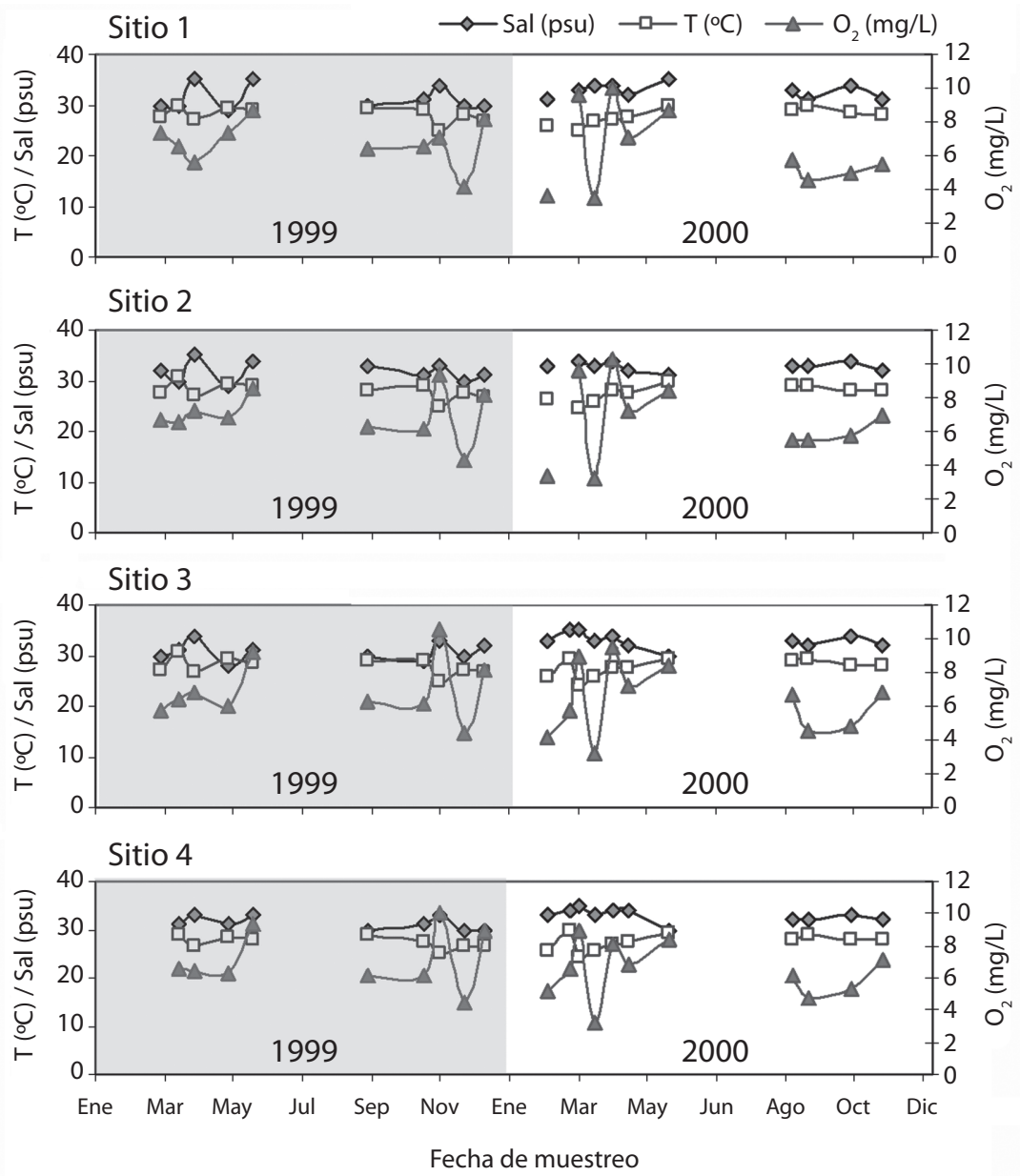

Fig. 2. Variación de los parámetros físico-químicos en la superficial, en los sitios de muestreo, Bahía Culebra, Pacífico norte de Costa Rica. $\mathrm{O}_{2}=$ Oxígeno disuelto en agua, $\mathrm{T}=$ Temperatura, $\mathrm{Sal}=$ Salinidad.

Fig. 2. Surface variations of physical and chemical parameters in sample sites, Bahía Culebra, North Pacific, Costa Rica. $\mathrm{O}_{2}=$ disolved oxygen in water, $\mathrm{T}=$ Temperature, $\mathrm{Sal}=$ Salinity.

se apreció mayor similitud entre los sitios de muestreo uno y cuatro (en la boca de la bahía y fuera de ella, respectivamente), mientras el sitio tres (con influencia estuarina) resultó ser la más disímil (Fig. 3).

\section{Parámetros biológicos}

Variación espacial: La mayor abundancia de mesozooplancton se presentó en la S1 (6 $216.90 \pm 5$ 125.26ind. $\left./ \mathrm{m}^{3}\right)$, seguido de la S2 (5 $016.93 \pm 3$ 829.43ind. $\left./ \mathrm{m}^{3}\right)$, S3 (4
$138.18 \pm 2892.80$ ind. $\left./ \mathrm{m}^{3}\right)$ y S4 (3 969.46 \pm 3 247.92ind. $/ \mathrm{m}^{3}$ ). Sin embargo, no se observaron diferencias significativas entre los sitios a lo largo del periodo de muestreo $(\mathrm{F}=0.927, \mathrm{p}=$ 0.431) (Fig. 4).

La relación de similitud (basado en un análisis de conglomerados) entre los datos de abundancia es consecuente con los datos obtenidos de los promedios generales. Así que se observó que la S3 y S4 son más similares entre sí que la S2 y S1, siendo este último el más disímil de los estudiados (Fig. 5). 


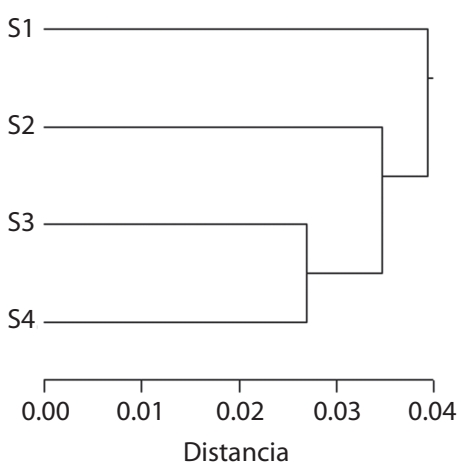

Salinidad

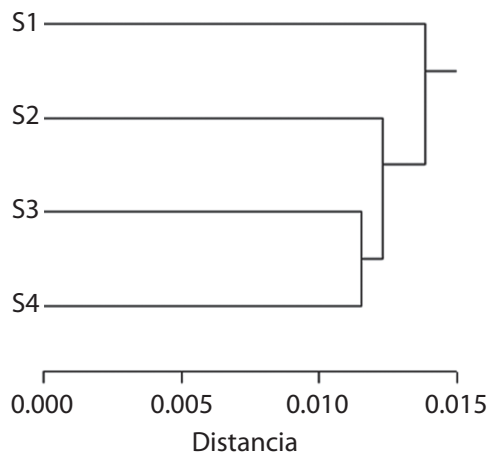

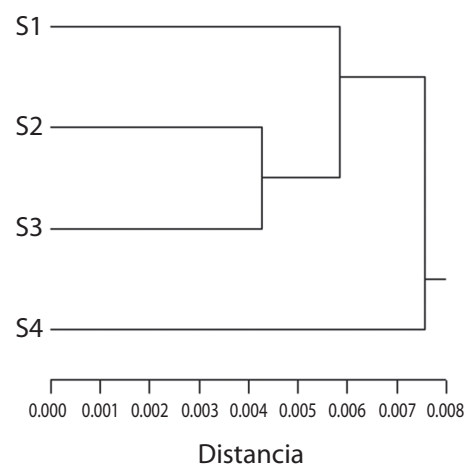

Disco de Secchi

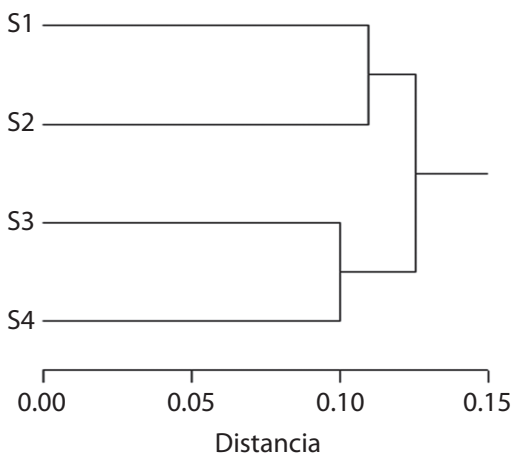

Fig. 3. Análisis de conglomerados de los parámetros físico-químicos: oxígeno disuelto $(\mathrm{mg} / \mathrm{l})$, temperatura $\left({ }^{\circ} \mathrm{C}\right)$, salinidad (PSU) y transparencia del agua (m).

Fig. 3. Cluster analysis of physical and chemical parameters: oxygen $(\mathrm{mg} / \mathrm{l})$, temperature $\left({ }^{\circ} \mathrm{C}\right)$, salinity $(\mathrm{PSU})$ and water transparency $(\mathrm{m})$.

Variación temporal: Se observó una gran dispersión de las abundancias de organismos con respecto a las fechas de muestreo $(\mathrm{F}=$ $4.954, \mathrm{p}=0.0001$ ). Aunque las diferencias en la abundancia no llegan a ser significativas entre 1999 y $2000(\mathrm{~F}=3.45, \mathrm{p}=0.060)$, se notó una disminución de la abundancia en el 2000 con respecto a 1999. Para 1999 se cuantificaron en promedio $5702.02 \pm 4$ 103.27ind. $/ \mathrm{m}^{3}$ (máx.= 16762.95 y mín. $\left.=785.20 \mathrm{ind} . / \mathrm{m}^{3}\right)$, mientras que para el 2000 el promedio fue de 4301.82 \pm 3 618.58ind. $/ \mathrm{m}^{3}$ (máx.= 19682.56 y mín. = 417.57ind. $/ \mathrm{m}^{3}$ ) (Fig. 6).
Se observó que durante la estación seca (diciembre-abril) la abundancia de mesozooplancton fue mayor que durante la estación lluviosa (mayo-noviembre), $(\mathrm{F}=6.588, \mathrm{p}=$ 0.012). Durante la época de secas, cuando se experimenta el evento del afloramiento costero, el promedio de abundancia fue 5441.12 土 3 946.97ind. $/ \mathrm{m}^{3}$ (máx= 19682.56 y mín.= $785.20 \mathrm{ind} . / \mathrm{m}^{3}$ ) y durante las estaciones lluviosas el promedio fue $3950.03 \pm 3730.84$ ind./ $\mathrm{m}^{3}$ (máx.= 16762.95 y mín. $=417.57$ ind. $/ \mathrm{m}^{3}$ ) (Fig. 7). Estos datos tomaron en consideración ambos años de estudio. 


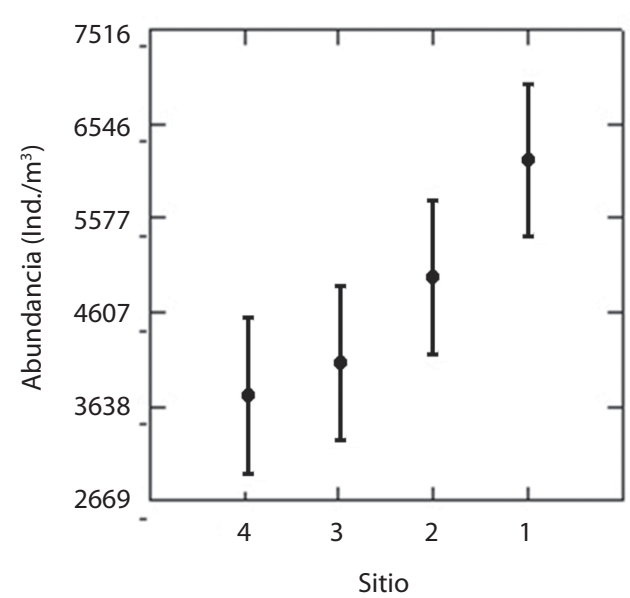

Fig. 4. Abundancia relativa del mesozooplancton por sitio de muestreo, Bahía Culebra, Golfo de Papagayo, 19992000.

Fig. 4. Relative abundances of mesozooplankton by sample sites, Bahía Culebra, Golfo de Papagayo, 1999-2000.

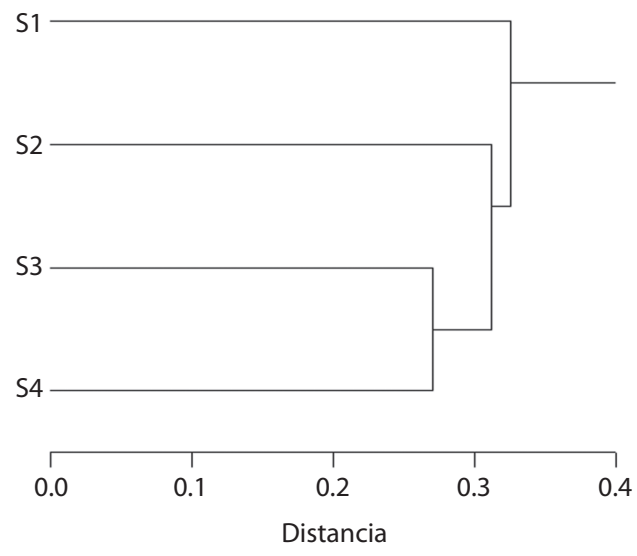

Fig. 5. Análisis de conglomerados para las abundancias en los sitios de muestreo (basado en el índice de similitud Bray and Curtis), Bahía Culebra, Golfo de Papagayo, 1999-2000.

Fig. 5. Cluster analysis of abundances in the sampling sites (based on Bray Curtis Index), at Bahía Culebra, Golfo de Papagayo, 1999-2000.

\section{Composición y variación estacional}

Un total de 26 taxones pertenecientes al mesozooplancton fueron identificados en los cuatro sitios de muestreo. Entre ellos cabe

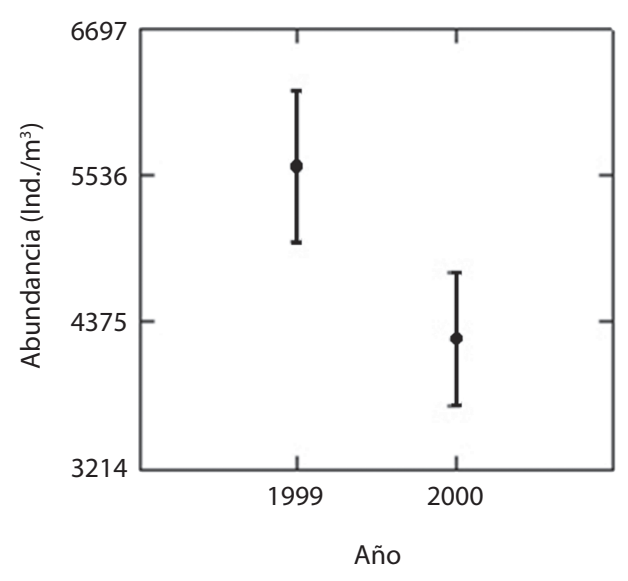

Fig. 6. Abundancia promedio de mesozooplancton (19992000), en Bahía Culebra.

Fig. 6. Average abundance of mesozooplankon (19992000), at Bahía Culebra.

destacar la presencia de los estadíos larvales de briozoarios, equinodermos (larvas ophiopluteus), braquiópodos, poliquetos, mysis, megalopas y de bivalvos, que forman parte del merozooplancton. Por otra parte, microcrustáceos como los copépodos, ostrácodos y cladóceros, así como las apendicularias, los quetognatos, sifonóforos y ctenóforos, fueron los grupos más representativos dentro del holozooplancton. Los grupos dominantes en abundancia fueron los microcrustáceos, especialmente los copépodos, con un promedio de $2358 \pm 2$ 290ind. $/ \mathrm{m}^{3}$, los ostrácodos, con 1 $084 \pm 1850 \mathrm{ind} . / \mathrm{m}^{3}$, los huevos de invertebrados, con un promedio de $844 \pm 7$ 069ind. $/ \mathrm{m}^{3}$, las apendicularias alcanzaron un promedio de $168 \pm 286$ ind. $/ \mathrm{m}^{3}$, mientras que las larvas de crustáceos tuvieron un promedio de $87 \pm 180$ ind. $/ \mathrm{m}^{3}$ y los cladóceros con un promedio de abundancia de $438 \pm 783$ ind. $/ \mathrm{m}^{3}$.

Los grupos más abundantes del mesozooplancton en Bahía Culebra durante el periodo de estudio mostraron una alta variabilidad con respecto a las fechas de muestreo (ANOVA, p < $0.05)$. De la misma forma, se observaron diferencias porcentuales en cuanto a su presencia a lo largo de los periodos anuales de estudio (1999 y 2000) (Fig. 7). Por ejemplo, aunque 

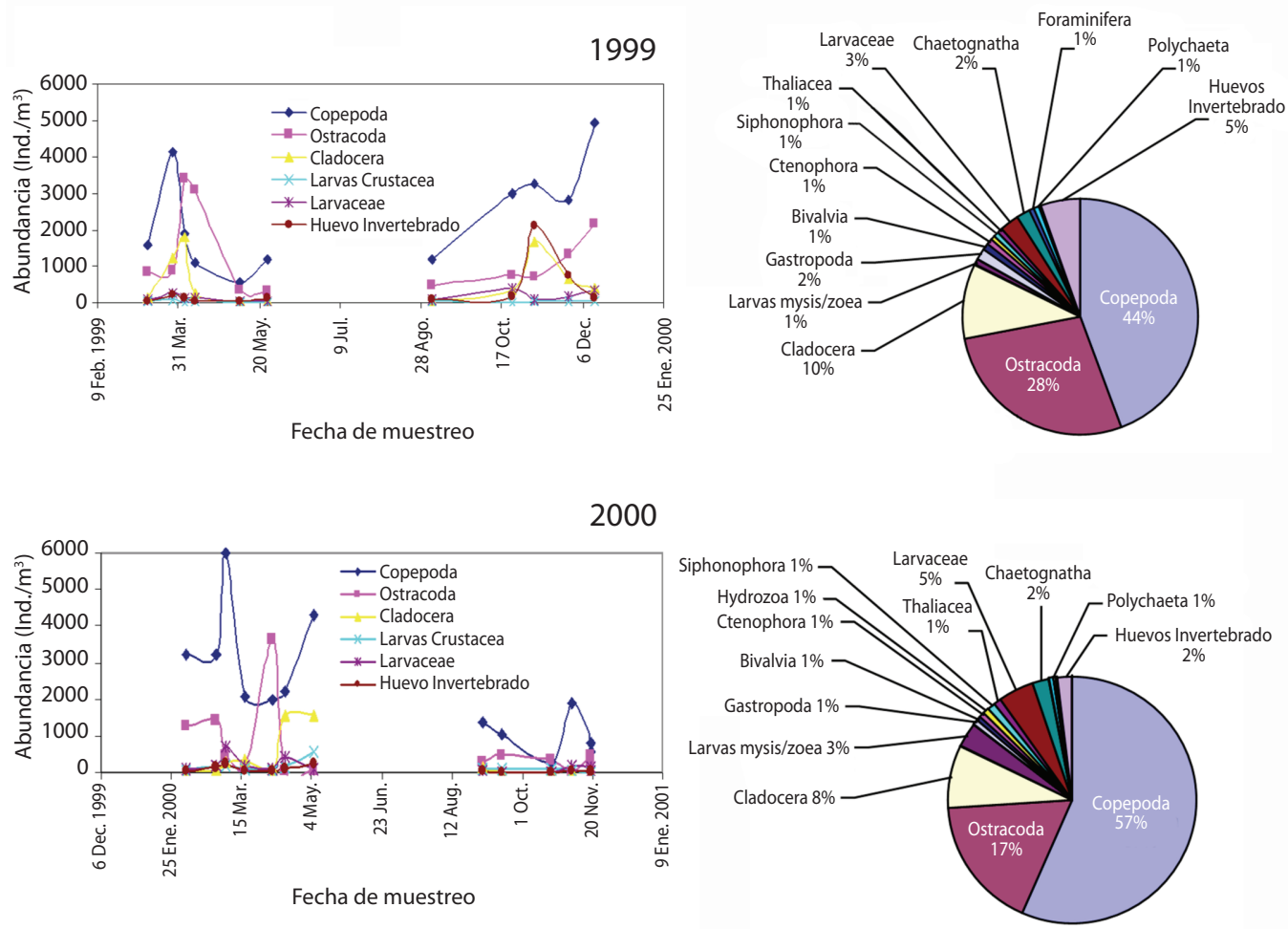

Fig. 7. Porcentajes de abundancia de los grupos taxonómicos de mesozooplancton durante 1999 y 2000, en Bahía Culebra, Golfo de Papagayo.

Fig. 7. Abundance percentages of mesozooplankton taxonomic groups during 1999-2000, at Bahía Culebra, Golfo de Papagayo.

los copépodos tuvieron una abundancia porcentual de un $44 \%$ en 1999 y $57 \%$ en el 2000 , también se observó que el promedio de abundancia relativa durante 1999 fue de 2555.82 \pm 2 141.37ind. $/ \mathrm{m}^{3}$ mientras en el 2000 su promedio de abundancia se redujo a $2199.92 \pm 2$ 413.67ind. $/ \mathrm{m}^{3}$, aun así estas diferencias no son estadísticamente significativas $(\mathrm{F}=1.609, \mathrm{p}=$ 0.208). De la misma forma, los ostrácodos se presentaron en un porcentaje significativamente mayor en 1999 (28\%) que en el 2000 (17\%) $(\mathrm{F}=12.56, \mathrm{p}=0.001)$ y ese mismo patrón siguió el componente de los cladóceros $(1999=8 \%$ y $2000=10 \%)(\mathrm{F}=9.27, \mathrm{p}=0.003)$, los huevos de invertebrados que pasaron de un $5 \%$ en 1999 a solamente un $2 \%$ en el $2000(\mathrm{~F}=14.59$, $\mathrm{p}=0.000)$ y aunque los moluscos planctónicos mostraron tener una baja abundancia en los dos años de estudio, la disminución observada de 1999 y con respecto al 2000 es significativa $(\mathrm{F}=$ $7.70, \mathrm{p}=0.007)$. El patrón inverso fue mostrado únicamente por las apendicularias, que pasaron de un promedio de $152.36 \pm 226.71$ ind. $/ \mathrm{m}^{3}$ en 1999 a $180.96 \pm 328.89$ ind.$/ \mathrm{m}^{3}$ en el $2000(\mathrm{~F}=$ $3.88, \mathrm{p}=0.05)$ y por las larvas de crustáceos, las cuales pasaron de un promedio de $43.34 \pm$ 61.37ind. $/ \mathrm{m}^{3}$ en 1999 a $123.28 \pm 230.33$ ind.$/ \mathrm{m}^{3}$ $(\mathrm{F}=8.420, \mathrm{p}=0.005)$.

\section{Variaciones espaciales}

Se observó que los grupos que alcanzaron mayor abundancia en los muestreos tienen diferencias significativas en cuanto a su presencia en los sitios de estudio. Los copépodos por 
ejemplo, presentaron una abundancia promedio mayor en la S1 que en el resto de sitios de muestreo $(\mathrm{F}=2.69, \mathrm{p}=0.05)$. Los ostrácodos mostraron un promedio de abundancia menor precisamente en la $\mathrm{S} 1(\mathrm{~F}=2.86, \mathrm{p}=0.04)$. En el caso de los moluscos planctónicos se observó que la abundancia menor se presentó en la S4 $(\mathrm{F}=3.164, \mathrm{p}=0.03)$. Otros grupos como el zooplancton gelatinoso, los cladóceros, poliquetos o quetognatos en el presente estudio, no llegan a tener abundancias con diferencias significativas entre los sitios ni muestran tendencias de predilección por alguno. Finalmente, algunos
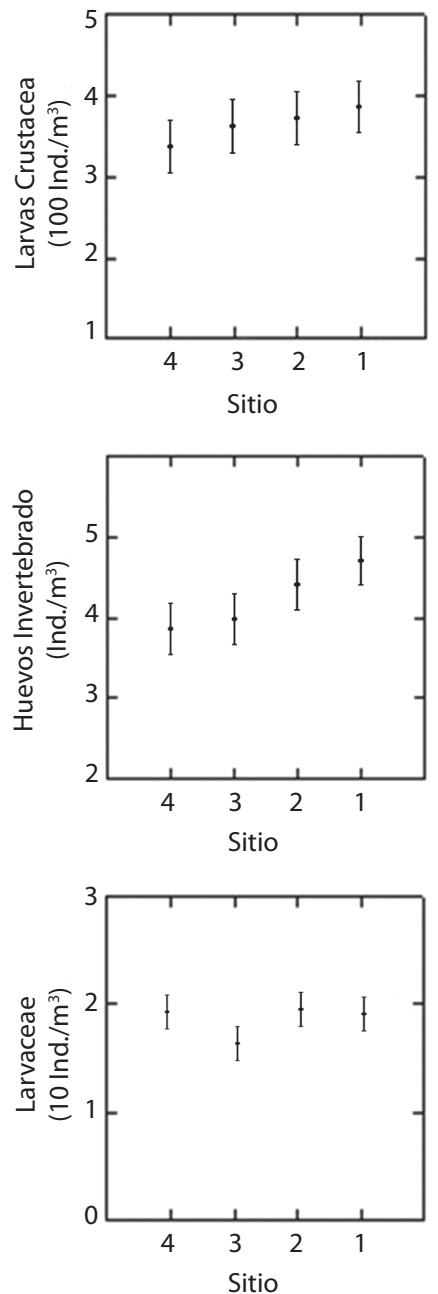

grupos no llegan a tener diferencias significativas entre los sitios pero muestran mayores abundancias en alguna específica, como el caso de los huevos de invertebrados, cuyo promedio de abundancia alcanzó su máximo en la $\mathrm{S} 1(\mathrm{~F}=$ $0.99, \mathrm{p}=0.402$ ), de la misma forma que las larvas de crustáceos $(\mathrm{F}=0.537, \mathrm{p}=0.66)($ Fig. 8).

\section{DISCUSIÓN}

Durante La Niña se encontraron mayores concentraciones de oxígeno y menores
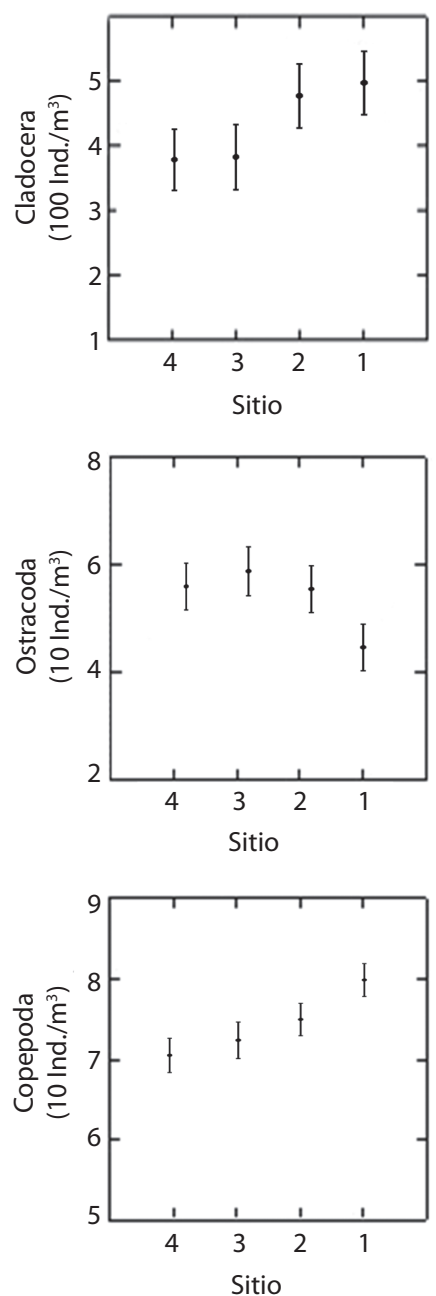

Fig. 8. Distribución espacial de la abundancia transformada de algunos grupos del mesozooplancton de Bahía Culebra, Golfo de Papagayo, 1999-2000.

Fig. 8. Spatial distribution of the transformed abundance of some mesozooplankton groups at Bahía Culebra, $1999-2000$. 
salinidades en el agua que son tendencias típicas de condiciones del fenómeno (siempre acompañados de los bajos valores de temperatura). Datos preliminares muestran que Bahía Culebra mantuvo durante 1997 y 1998, a través del periodo de afloramiento, promedios en la temperatura de $29.8 \pm 1.1^{\circ} \mathrm{C}$, sin embargo, la intensidad y la permanencia de las masas de agua fueron variables (Jiménez et al. 2001). Si se comparan los promedios de temperatura de estos años, declarados bajo la influencia del fenómeno de El Niño (NOAA 2003) con los obtenidos durante 1999 y 2000 del presente estudio $\left(27.84 \pm 1.59\right.$ y $27.66 \pm 1.49^{\circ} \mathrm{C}$, respectivamente) se nota un importante descenso en la temperatura de por lo menos $2^{\circ} \mathrm{C}$. A nivel regional, el Golfo de Papagayo experimentó un descenso de la temperatura de por lo menos $1^{\circ} \mathrm{C}$ por debajo de lo normal en el Pacífico Ecuatorial durante el primer trimestre de 1999 (Coen et al. 2001). Para los últimos meses de 1999 este año, se dio una transición paulatina del episodio frío y para el segundo semestre del 2000, se llegaba a un estado "transicional a normal" del mismo (Coen et al. 2001).

Las mayores concentraciones del mesozooplancton de Bahía Culebra en 1999 coinciden con las altas concentraciones promedio oxígeno disuelto y las menores de salinidad. También coinciden con importantes florecimientos de clorofila a detectados de forma regional en el Golfo de Papagayo, especialmente durante el primer semestre de 1999 (Coen et al. 2001). Para el 2000, se aprecia la importante disminución de la abundancia de zooplancton en general, coincidiendo de nuevo con el retorno a la "normalidad" de las condiciones oceanográficas. Sin embargo, la carencia de datos previos del comportamiento de la abundancia durante El Niño y años "normales" en Bahía Culebra limita las conclusiones acerca de los resultados obtenidos en la presente investigación.

Todos los grupos (con excepción de las apendicularias y las larvas de crustáceos) muestran una mayor abundancia durante 1999, para luego llegar a bajas abundancias en el 2000. La mayor abundancia de ostrácodos (10\% de la abundancia total para 1999) se asocia con el promedio de baja salinidad para ese mismo año, mientras que un detrimento en la abundancia $(8 \%)$ se presenta el siguiente año, cuando la salinidad promedio es mayor. Este patrón ha sido observado previamente (Calef \& Grice 1967) y se atribuye a la afinidad costera (o con influencia estuarina) que tienen estos microcrustáceos. Con respecto al patrón de mayor abundancia en el 2000 con respecto a 1999 observado en las apendicularias, es inesperado, debido a que estos urocordados incrementan rápidamente (en cuestión de meses) sus poblaciones después de eventos de El Niño (Lavaniegos \& López 1997), debido a su corto tiempo de generación y sus hábitos alimentarios como filtradores de partículas finas (Acuña \& Anadón 1992, Gasca \& Suárez 1996, MoralesRamírez \& Nowaczyck 2006). Los resultados del presente estudio concuerdan con otros trabajos donde se ha logrado asociar masas de agua con baja salinidad y alta concentración de oxígeno (además de temperatura) con ricas concentraciones de zooplancton (Roesler \& Chelton 1987).

Se encontró que la mayor abundancia de zooplancton es durante la estación seca, la cual se espera por su relación con los pulsos de afloramiento costero que se presentan en esta zona. Este mismo patrón fue observado por Bednarsky (2001) para el macrozooplancton de Bahía Culebra, donde fueron encontrados, además, como géneros dominantes de copépodos Eucalanus, Centropages, Temora y Acartia. Según este estudio, la composición de copépodos encontrada en Bahía Culebra es típica de sistemas de afloramiento.

Durante las épocas lluviosas estudiadas, las abundancias de mesozooplancton en las diferentes sitios de muestreo también mostraron algunos valores altos que podrían estar relacionadas con aportes de materia orgánica vía aguas pluviales que entran a la bahía por riachuelos y esteros o a procesos reproductivos de algunas especies residentes (como cangrejos, estomatópodos, gastrópodos y otros). Los nutrimentos que serían aportados por el afloramiento mismo o por vías pluviales, podrían ser aprovechados en cuestión de semanas en 
la bahía por pequeños heterótrofos (ciliados, bacterias y otros protistas) así como por el fitoplancton. Estos grupos serían aprovechadas por el zooplancton herbívoro y omnívoro (meso y macrozooplancton), para ser posteriormente presas del zooplancton carnívoro, todo esto en lapsos relativamente cortos.

Las abundancias porcentuales de los grupos dominantes como microcrustáceos, quetognatos y tunicados, son cercanos a los reportados para el Pacífico Tropical Oriental (Longhurst 1985), pero en esta bahía se presenta un importante aporte de formas meropláncticas más frecuentes en zonas donde se desarrollan arrecifes coralinos o ambientes estuarinos. Este aporte puede ser debido a la presencia de una de las comunidades arrecifales más importantes del Pacífico costarricense (Cortés \& Jiménez 2003) y la representación de microambientes estuarinos en por lo menos ocho esteros con vegetación típica de manglar (Córdoba et al. 1998, Zamora-Trejos \& Cortés 2009). Con todo esto, se logra corroborar que Bahía Culebra, al igual que otras bahías tropicales poco profundas, muestra una alta variabilidad en las condiciones físico-químicas que probablemente condicionan el desarrollo de las comunidades zoopláncticas (Ríos-Jara 1998).

Las relaciones espaciales que se observaron en la bahía son también interesantes. Los cladóceros, por ejemplo, muestran cierta predilección por las partes internas de la bahía, que son los sitios menos profundos y con influencia estuarina. Este patrón ha sido observado en zonas dinámicamente diferentes de Costa Rica, como el Golfo Dulce, aunque con las mismas características de profundidad y circulación estuarina (Quesada-Alpízar 2001). Se supone que estas condiciones favorecerían el ciclo reproductivo de este grupo, en el que todas sus especies muestran estadíos de reposo bentónicos (Raymont 1983, Longhurst 1985). Sin embargo, esta relación con zonas estuarinas puede estar relacionada a las especies cuyos hábitos alimentarios se basan en la filtración de partículas finas en suspensión (Ruppert \& Barnes 1996).
Asímismo, las formas larvales de crustáceos y los huevos de invertebrados ocupan preferentemente el sitio de carácter más estuarino y poca profundidad de la bahía, denotando la importancia de los sitios cercanos a los esteros como zonas de desove, crianza y hábitat de macroinvertebrados móviles (como cangrejos y camarones) y sésiles (e.j. braquiópodos, cirripedios, colonias hidroides. No es de extrañar tampoco que las mayores concentraciones de mesozooplancton se encuentren asociadas a estas zonas pues son considerados como los ecosistemas más productivos del planeta en cuanto a productividad primaria y secundaria (Caspers 1967, Thurman 1996).

La abundancia promedio de los ostrácodos fue más baja en la $\mathrm{S} 1$ (menos profundo y con influencia estuarina) que el resto de los sitios de muestreo, contrariamente con el patrón encontrado para los copépodos, pues es en este sitio donde muestran su mayor abundancia promedio. Las diferentes especies de ostrácodos pueden tener hábitos alimentarios tan variados como comensales, carnívoros, carroñeros y filtradores. El caso presentado en Bahía Culebra sugiere, por una parte, un posible efecto de la depredación de los ostrácodos sobre los copépodos (si las dos especies presentes en este sitio fueran formas carnívoras), pues en otras regiones se ha observado especies como Macrocypridina, alimentándose activamente de muchos copépodos (Gasca y Suárez 1996), sin embargo, también podría relacionarse este patrón con una exclusión competitiva entre las especies, si en ambos grupos (copépodos y ostrácodos) se presentaran hábitos alimentarios semejantes. Esta hipótesis se fortalece si se deduce que el hábito probable de los grupos es la alimentación por filtración de materia en suspensión.

Este estudio aporta nuevos conocimientos de los cambios a meso escala en la comunidad del mesozooplancton marino de Bahía Culebra, y macro escala con los cambios observados durante La Niña de 1999. Sin embargo, quedan algunas preguntas pendientes de responder con respecto a Bahía Culebra y el zooplancton en general. Se sugieren algunas de las ideas 
para futuras investigaciones las cuales podrían incluir desde estudios de monitoreo físico químico y biológico hasta aspectos socioeconómicos de las comunidades humanas que hacen uso de los recursos marino-costeros de esta zona. De la misma forma es importante tomar en consideración los planes de desarrollo que a nivel estatal se tienen para esta zona y proveer los impactos potenciales sobre los recursos y ecosistemas del área que ese desarrollo puede provocar: A) Establecer un programa de monitoreo continuo que logre generar información para todos los meses del año, especialmente para poder entender la dinámica del zooplancton durante años sin eventos como El Niño o La Niña, y además para complementar la información presentada en este informe, especialmente en los meses que no fue posible muestrear. B) Nuevas investigaciones deben tomar en consideración la posible presencia de zooplancton demersal (asociado a las comunidades coralinas de esta bahía), para lo cual se sugiere tomar en consideración la posibilidad técnica de realizar muestreos nocturnos. Esta estrategia también coadyuvará en una estimación más certera de la abundancia, la composición así como de la biomasa en horas donde teóricamente, los grupos se encuentran de una forma significativamente mayor en la columna de agua, sea para alimentarse o para reproducirse. C) Es urgente incentivar la toma de decisiones, claras y efectivas, de protección a los esteros en la zona, ya que se ha demostrado en este estudio que sitios cercanos a estas zonas de humedal (representados por el estero Iguanita) tienen una mayor abundancia de varios grupos o de larvas con respecto al resto de los sitios, por lo tanto resultan ser importantes zonas de crianza de crustáceos y otros invertebrados marinos. D) El control de la calidad de las aguas que por escorrentía superficial llegan a la bahía debe ser extremo, ya que enriquecimientos por materia orgánica pueden alterar la composición y abundancia del zooplancton y a su vez afectar a otros organismos de niveles tróficos superiores, como son los peces de interés biológico y comercial y mamíferos marinos. E) El planeamiento de estructuras artificiales como diques, muelles, marinas debe tomar en cuenta la presencia de organismos sensibles a cambios en las condiciones de las masas de agua como son los ostrácodos y las apendicularias, ya que son grupos de abundancia relativamente alta dentro de la bahía. De la misma forma se debe tomar en cuenta la presencia de muchas especies de zooplancton gelatinoso, en el cual se incluyen organismos que han sido denominados plagas en bahías donde la construcción de diques ha cambiado el patrón de circulación normal de las aguas o en zonas donde han introducido especies por medio de las aguas de balastre ya que llegan a alterar el balance natural de las comunidades planctónicas que se desarrollan en una estación determinado.

\section{AGRADECIMIENTOS}

Deseamos agradecer a Eleazar Ruiz su ayuda en el trabajo de campo. Este artículo es una contribución del proyecto 808-99236 "Ecología del zooplancton Bahía Culebra, Golfo de Papagayo, con énfasis en los copépodos y zooplancton gelatinoso" desarrollado bajo la responsabilidad del segundo autor y financiado por la Vicerrectoría de Investigación de la Universidad de Costa Rica. Formó parte de los requisitos para el grado de M.Sc. en la Universidad de Costa Rica por parte de la primera autora.

\section{RESUMEN}

Se estudian los patrones anuales de la composición, abundancia y distribución espacial-temporal de mesozooplancton en la zona de afloramiento de Bahía Culebra, en la costa norte del Pacífico de Costa Rica. Bahía Culebra presenta una alta variabilidad en las condiciones físico-químicas y se encontraron 26 grupos de mesozooplancton (meroplanctónicas y holoplanctónicos). Los copépodos (2 $358.53 \pm 2$ 290.90ind. $/ \mathrm{m}^{3}$ ), ostrácodos (1 $\left.084.46 \pm 1850.20 \mathrm{ind} . / \mathrm{m}^{3}\right)$ y huevos de invertebrados (844.26 $\left.\pm 7069.27 \mathrm{ind} . / \mathrm{m}^{3}\right)$ fueron los grupos dominantes. Hubo mayores abundancias en 1999 que en el 2000, con la excepción de apendicularias y larvas de crustáceos. El zooplancton fue más abundante en la época seca de ambos años. Las estaciones de la boca muestran un flujo oceánico de formas holoplanctónicos, mientras que el interior de la bahía meroplanctónicas fueron importantes las formas. La 
abundancia y distribución del mesozooplancton parecen reflejar los pulsos de surgencia costera.

Palabras clave: Mesozooplancton, surgencias, La Niña, Pacífico Oriental, Golfo de Papagayo, Costa Rica, Bahía Culebra.

\section{REFERENCIAS}

Acuña, J.L. \& R. Anadón. 1992. Appendicualrian assemblages in a shelf area and their relationship with temperature. J. Plank. Res. 14: 1233-1250.

Alvariño, A. 1972. Zooplancton del Caribe, Golfo de México y regiones adyacentes del Pacífico. Mem. IV Congr. Nacl. Oceanogr., México: 223-247.

Anónimo. 2001. Papagayo: el proyecto que terminará de situar a Costa Rica en el mapamundi turístico. Boletín Divulgativo, Insituto Costarricense de Turismo. San José, Costa Rica.

Bednarski, M. 2001. Macrozooplankton of Culebra Bay, Costa Rica, with an emphasis on copepods. Tesis M.Sc., Univ Bremen. Bremen, Alemania.

Bednarski, M. \& A. Morales-Ramírez. 2004 Composition, abundance and distribution of macrozooplankton in Culebra Bay, Gulf of Papagayo, Pacific coast of Costa Rica and its value as bioindicators of pollution. Rev. Biol. Trop. 53 (Supl. 2): 105-118.

Bianchi, G. 1991. Demersal assemblages of the continental shelf and slope edge between the Gulf of Tehuantepec (México) and the Gulf of Papagayo (Costa Rica). Mar. Ecol. Prog. Ser. 73: 121-140.

Bougis, P. 1976. Marine Plankton Ecology. North Holland Publ., Amsterdam.

Brenes, C., B. Kwiecinski, L. D’Croz \& J. Chaves. 1995. Características oceanográficas de la plataforma Pacífica de América Central y aguas ayacentes. Informe Técnico para PRADEPESCA. UNA, Heredia.

Brugnoli-Olivera, E. \& A. Morales-Ramírez. 2008. An aproximation on the grazing pressure of three species of copepods and their influence in the natural phytoplankton populations structure in Gulf of Nicoya, a tropical estuary, Pacific coast of Costa Rica. Rev. Biol. Mar. Oceanogr. 43: 75-89.

Brugnoli-Olivera, E., E. Díaz-Ferguson, M. DelfinoMachin, A. Morales-Ramírez \& A. Dominici-Arosemena. 2004. Composition of the zooplankton community, with emphasis in copepods, in Punta Morales, Golfo de Nicoya, Costa Rica. Rev. Biol. Trop. 52: 897-902
Calef, G. \& G. Grice. 1967. Influence of the Amazon River outflow on the ecology of the western tropical Atlantic. J. Mar. Res. 25: 84-04.

Caspers, H. 1967. Estuaries: analysis of definitions and biological considerations, p: 6-8. In G. Lauff (ed.). Estuaries. Amer. Assoc. Adv. Sci., Washington, D.C.

Clarke, K. \& R. Warwick. 1994. Change in Marine Communities: An Approach to Statistical Analysis and Interpretation. Nat. Envir. Res. Council, Reino Unido

Coen, E., C. Brenes \& D. Ballesteros. 2001. El Niño, La Niña y los afloramientos en el Pacífico Tropical Oriental. Ambientico 94: 7-8.

Córdoba, R., J. Romero, N. Windevoxhel, E. Madrigal, J. Bravo, P. Cordero \& L. Rivera. 1998. Inventario de los humedales de Costa Rica. UICN, San José, Costa Rica.

Cortés, J. \& C.E. Jiménez. 2003. Corals and coral reefs of the Pacific of Costa Rica: history, research and status, p. 361-385. In J. Cortés (Ed.). Latin American Coral Reefs. Elsevier Science, Amsterdam.

Desrosiers, C., H. Hartmann \& A. Morales-Ramírez. 2007. Dinámica estacional de la maraña alimentaria (bacterio-nano y microplancton) en Bahía Culebra, Golfo de Papagayo, costa Pacífica de Costa Rica. XI COLACMAR, Florianópolis, Brasil. Abstract.

Dominici-Arosemena, A., E. Brugnoli-Olivera, J. CortésNúñez, H. Molina-Ureña \& M. Quesada-Alpízar. 2005. Community structure of eastern Pacific reef fishes (Gulf of Papagayo, Costa Rica). Tecnociencia 7: 19-41.

Estrada, E. 2003. Zooplankton dynamics in the Golfo Dulce, Costa Rica: feeding preferences and grazing rates in main groups of macrocrustaceans. Tesis M.Sc., Univ. Bremen, Bremen, Alemania.

Fiedler, P., V. Philbrick \& F. Chávez. 1991. Oceanic upwelling and productivity in the eastern tropical Pacific. Limnol. Oceanogr. 36: 1834-1850.

Gasca, R. \& E. Suárez. 1996. Introducción al estudio del zooplancton marino. ECOSUR/CONACIT, Quintana Roo, México.

Guzmán, H.M. \& V.L. Obando. 1988. Diversidad y abundancia diaria y estacional del zooplancton marino de la Isla del Caño, Costa Rica. Rev. Biol. Trop. 36: 139-150.

Irigoien, X., J. Huisman \& R.P. Harris. 2004. Global diversity patterns of marine phytoplankton and zooplankton. Nature 429: 863-867. 
Jiménez, C.E. 1997. Corals and coral reefs of Culebra Bay, Pacific coast of Costa Rica: Anarchy in the reef. Proc. 8th Int. Coral Reef Symp., Panamá 1: 329-334.

Jiménez, C. 1998. Arrecifes y comunidades coralinas de Bahía Culebra, Pacífico Norte de Costa Rica (Golfo de Papagayo): Aspectos ecológicos. Tesis M.Sc., Univ. Costa Rica, San Pedro, San José.

Jiménez, C. 2001a. Seawater temperature measured at the surface and at two depths (7 and $12 \mathrm{~m}$ ) in one coral reef at Culebra Bay, Gulf of Papagayo, Costa Rica. Rev. Biol. Trop. 49 (Supl. 2): 153-161.

Jiménez, C. 2001b. Arrecifes y ambientes coralinos de Bahía Culebra, Pacífico de Costa Rica: aspectos biológicos, económico-recreativos y de manejo. Rev. Biol. Trop. 49 (Supl. 2): 215-231.

Jiménez, C., J. Cortés, A. León \& E. Ruiz. 2001. Coral bleaching and mortality associated with the 1997 1998 El Niño in an upwelling environment in the eastern Pacific (Gulf of Papagayo, Costa Rica). Bull. Mar. Sci. 69: 151-169.

Jiménez, C., G. Bassey, Á. Segura \& J. Cortés. 2010. Characterization of the coral communities and reefs of two previously undescribed locations in the upwelling region of Gulf of Papagayo (Costa Rica). Rev. Cienc. Mar. Cost. 2: 95-108.

Lavaniegos, B. \& D. López. 1997. Fatty acid composition and community structure of plankton from San Lorenzo Channel, Gulf of California. Est. Coast. Shelf Sci. 45: 845-854.

Lavaniegos, B., L. Jiménez-Pérez \& G. Gaxiola. 2002. Plankton response to El Niño 1997-1998 and La Niña 1999 in the southern region of the California Current. Prog. Oceanogr. 54: 33-58.

Lenz, J. 2000. Introduction, p. 1-30. In R. Harris, P. Wiebe, J. Lenz, H. Skjodal \& M. Huntley (eds). Zooplankton Methodology Manual. Academic, Londres.

Longhurst, A. 1985. Relationships between diversity and vertical structure of the upper ocean. Deep Sea Res. 32: $1535-1570$.

Morales-Ramírez, A. 1996. Checklist of copepods from Gulf of Nicoya, Coronado Bay and Golfo Dulce, Pacific coast of Costa Rica, with comments on their distribution. Rev. Biol. Trop. 44 (Supl. 3): 103-113.

Morales-Ramírez, A. 2001. Biodiversidad marina de Costa Rica, los microcrustaceos: Subclase Copepoda (Crustacea: Maxillipoda). Rev. Biol. Trop. 49 (Supl. 2): 115-133.
Morales-Ramírez, A. 2008. Caracterización cualitativa del zooplancton del Área de Conservación Marina Isla del Coco (ACMIC), Océano Pacífico de Costa Rica. Rev. Biol. Trop. 56 (Supl. 2): 159-169

Morales-Ramírez, A. \& E. Brugnoli-Olivera. 2001. El Niño 1997-1998 impact on the plankton dynamics in the Gulf of Nicoya, Pacific coast of Costa Rica. Rev. Biol. Trop. 49 (Supl. 2): 103-114.

Morales-Ramírez, A. \& J. Jacob. 2008. Seasonal vertical distribution, abundance, biomass and biometrical relationships of ostracods in Golfo Dulce, Pacific coast of Costa Rica. Rev. Biol. Trop. 56 (Supl. 4): $125-147$

Morales-Ramírez, A. \& J. Nowaczyk. 2006. El zooplancton gelatinoso del Golfo Dulce, Pacífico de Costa Rica, durante la transición de la estación lluviosa a la seca 1997-1998. Rev. Biol. Trop. 54 (Supl. 1): 201-223

Morales-Ramírez, A. \& J.A. Vargas-Zamora. 1995. Especies comunes de copépodos pelágicos (Crustacea: Copepoda) del Golfo de Nicoya. Costa Rica. Rev. Biol. Trop. 43: 207-218.

Muller-Parker, G. \& J. Cortés. 2001. Spatial distribution of light and nutrients in some coral reefs of Costa Rica. Rev. Biol. Trop. 49 (Supl. 2): 251-263.

NOAA. 1979. Oceanic and related atmospheric phenomena as viewed from environmental satellites. National Environmental Satellite Service, NOAA, Washington D.C.

NOAA. 2003. National Oceanographic and Atmospheric Agency. http://www.noaa. gov/

Quesada-Alpízar, M.A. 2001. Distribución vertical y variación temporal y espacial de la biomasa del zooplancton en el Golfo Dulce durante el fenómeno de El Niño 1997-1998. Tesis M.Sc., Univ Costa Rica, San Pedro, Costa Rica.

Quesada-Alpízar, M.A. \& A. Morales-Ramírez. 2006. Posible efecto de El Niño en el zooplancton no gelatinoso del Golfo Dulce, Pacífico de costa Rica, 1997 1998. Rev. Biol. Trop. (Supl. 1): 225-240.

Raymont, J. 1983. Plankton and Productivity in the Oceans: Zooplankton (Vol. 2). 2nd. ed. Pergamon Press, Oxford y Londres.

Ríos-Jara, E. 1998. Spatial and temporal variations in the zooplankton community of Phosphorescent Bay, Puerto Rico. Est. Coast. Shelf Sci. 46: 797-809.

Rodríguez-Sáenz, K.E. 2005. Distribución espacial y temporal de la biomasa, composición y abundancia del zooplancton, con énfasis en hidromedusas de Bahía 
Culebra, durante La Niña 1999 y el 2000. Tesis M.Sc., Univ. Costa Rica, San Pedro, Costa Rica.

Rodríguez-Sáenz, K. \& R. Gasca 2009. Siphonopheres, Texto: 151-156, Lista especies CD: 151-156. In I.S. Wehrtmann \& J. Cortés (eds). Marine Biodiversity of Costa Rica, Central America. Monogr. Biol. 86. Springer, Berlín.

Rodríguez-Saénz, K. \& J. Rodríguez-Fonseca. 2004. Avistamientos del delfín manchado, Stenella attenuata (Cetacea: Delphinidae) en Bahía Culebra, Costa Rica, 1999-2000. Rev. Biol. Trop. 52 (Supl. 2): 189-194.

Roesler, C \& D. Chelton. 1987. Zooplankton variability in the California Current, 1951-1982. Calif. Coop. Ocean. Fish. Investig. Rep. 28: 59-96.

Ruppert, E. \& R. Barnes. 1996. Zoología de los Invertebrados. 6ta Ed. McGraw-Hill, México.
Steedman, H. 1976. Zooplankton Fixation and Preservation. Monogr. Oceanogr. Method. 4. UNESCO, Paris.

Suárez-Morales, E. \& E. Rivera-Arriaga. 1998. Zooplancton e hidrodinámica en zonas litorales y arrecifales de Quintana Roo, México. Hidrobiológica 8: 19-32.

Thurman, H. 1996. Essentials of Oceanography. Prentice may, New Jersey, EUA.

von Wangelin, M. \& M. Wolff. 1996. Comparative biomass spectra and species composition of zooplankton communities in Golfo Dulce and Golfo Nicoya, pacific coast of Costa Rica. Rev. Biol. Trop. 44 (Supl.3): 135-155

Zamora-Trejos, P. \& J. Cortés. 2009. Los manglares de Costa Rica: Pacífico norte. Rev. Biol. Trop. 57: 473-488. 
